\title{
Ce que William Ritter n’a pas appris à Le Corbusier
}

\author{
Xavier GaLmiche \\ Sorbonne Université, Faculté des Lettres, UMR Eur'ORBEM (8224)
}

\begin{abstract}
William Ritter fut un touche-à-tout, parfois un dilettante. Souvent laissée en plan, la production de ce graphomane voyageur et critique péremptoire compose un kaléidoscope oublié sur lequel il vaut la peine de coller son œil : car il imposa, avec une franchise qui allait parfois jusqu'à l'ingénuité, certaines dissonances dans la culture helvétique et en général francophone de la « fin de siècle ". On s'arrête ici sur celles qui le lancèrent vers des horizons méconnus, des pays des Balkans puis de l'Europe centrale, et le poussèrent à les évoquer d'une plume singulière qui lui permit de cultiver ses différences, entre autres son amour des garçons. Il s'inscrit donc trois fois dans la culture 1900 : dans l'histoire des rapports interculturels, du style, mais aussi de l'éveil de la question des « genres » et de la culture homosexuelle.

Keywords: écrivain voyageur, «fin de siècle », Balkans, Europe centrale, homosexualité
\end{abstract}

\section{Le kaléidoscope oublié}

\section{Le syndrome d'Eckermann}

On connaît le nom du « docteur » Johann Peter Eckermann grâce à ses conversations avec Goethe, qu'il consigna scrupuleusement et édita en I836. Mais de son œuvre poétique personnelle, qui avait su attirer la bienveillance du maître, mais de ses goûts de lettré weimarien, poussé dans le monde académique par la protection de son glorieux ami, on ignore presque tout. Il y a un peu d'Eckermann dans la figure de William Ritter. L'obscurité qui entoure son œuvre " aujourd'huy " (c'est ainsi qu'il aurait orthographié : ce style précieux et décadent fait partie des éléments qui compliquent d'emblée la lecture contemporaine de ses textes) ne semble se dissiper que grâce à la réputation d'hommes célèbres : dans les cantons francophones de Suisse, son père Guillaume, l'ingénieur (Bauermeister); et dans le vaste monde, des figures dont il sut devenir le familier et surtout le correspondant. Les deux noms internationalement les plus médiatiques sont incontestablement Gustav Mahler et Le Corbusier. Symptomatiquement, ils représentent les deux mondes de référence de Ritter, la Suisse et l'Europe centrale (au sens large, évidemment) d'avant le séisme de la Première Guerre mondiale. C'est à Munich que Ritter découvrit en I9or l'œuvre de Mahler dont il suivit, jusqu'à la mort de ce dernier en I9II, l'évolution depuis ses différents lieux de résidence (Prague et la Bavière surtout); c'est aussi à Munich que le jeune Charles-Édouard Jeanneret sut le trouver en I9IO, suivant un principe d'attraction des compatriotes à l'étranger dont Ritter lui-même, quelque vingt 
ans auparavant, avait profité (Le Corbusier-Ritter 20I4). Il entreprit en effet son premier voyage à l'Est pour rendre visite à des Neuchâtelois installés à Bucarest, et s'il trouva des raisons bien personnelles de succomber aux charmes de la Roumanie, puis, à la suite, des Monténégro, Bohême, pays slovaque, etc., la prime impulsion est venue du phénomène sociologique discret mais constant d'une émigration helvétique vers les régions d'Europe centrale et des Balkans (Michaud 2008). Mais de l'autre côté, le futur «Corbu » est du même pays que Ritter, au nord du lac de Neuchâtel, et ils s'y retrouveront lorsque William s'y sera retiré, ayant en hâte quitté l'Allemagne à l'aube de la guerre.

À cette glorieuse paire il conviendrait d'ajouter une petite liste d'auteurs dont Ritter fut le proche, et le correspondant plus ou moins régulier. Il s'agit exclusivement de peintres, célébrissimes en leur pays respectif mais dont le renom à l'étranger dépend beaucoup du poids géopolitique de ce dernier et donc de ses capacités à exporter sa culture. Ce qui donne à peu près, par ordre de notoriété, le Polonais Józef Mehoffer (Bourgarel et al. 1995), le Roumain Nicolae Grigorescu (Vlasiu 2008), le Tchèque ou plutôt le Morave Joža Úprka (Ritter I9I2 ; Galmiche-Theinhardt 20II), le Slovaque Martin Benka'.

Les correspondances furent à William ce que les conversations avec Goethe étaient à Eckermann. Un soin particulier entourait les lettres reçues, mais aussi les lettres envoyées : en graphomane névrosé, Ritter rédigeait sur des « copies-lettres », livres de correspondances tels qu'en possédaient les commis aux écritures des artisans et des marchands, dont les pages sont doublées afin de conserver un calque de la lettre, ce qui permet à l'expéditeur de garder une copie. Cette grande méticulosité, William sut en contaminer Josef Červ, un de ses amants et son dernier compagnon, qui devint en 1933 son fils adoptif, sa canne de vieillesse, et, donc, en francisant l'orthographe de son nom (Tcherv), son archiviste. Tant d'attentions continuées finirent par porter leur fruit : l'édition de la belle correspondance Ritter-Mahler par Claude Meylan a été le premier ouvrage à initier, après quelques études isolées², l'entreprise d'évaluation critique de Ritter mélomane (Meylan 200o) ; quant à celle avec Le Corbusier, elle occupe un volume de l'édition menée à bien par Marie-Jeanne Dumont, des correspondances majeures du jeune Jeanneret (Le Corbusier-Ritter 20I4 déjà cité). Il faut compter avec le vaste archipel d'autres « égo-documents », principalement conservés par les ALS : le journal, que Ritter a tenu depuis ses quatorze ans, en I88I, jusqu'à sa mort (l'inventaire provisoire établi pour les ALS de Berne en a répertorié 287 ca-

\footnotetext{
I La volumineuse monographie écrite par Ritter sur Benka entre I937 et I947 est restée à l'état de manuscrit (ALS, Fonds WR, boite 44).

2 Voir la bibliographie ici en fin d'article ; notons que le nom de Ritter, qui servit de son vivant de caution à des causes nationales exclusives, a été l'étiquette de publications suisses associées au retour du nationalisme violent à la fin du Xx $x^{\mathrm{e}}$ siècle (Kirschbaum, Mikuš).
} 
hiers de divers formats), qui s'entrecroisent avec des calepins de voyage dont les qualités littéraires ont poussé certains optimistes à risquer des publications partielles - édition d'échantillons, Galmiche (2019 : I35-I49; ou de fragments, Wahlen 20I6) et des carnets de dessins, qui fournissent une importante moisson de documents (relevés ethnographiques et/ou instantanés glanés au hasard des rencontres). Cette masse documentaire se prolonge par l'œuvre graphique et s'est encore agrandie depuis que la Bibliothèque publique et universitaire de Neuchâtel (BPUN) a procédé à la restauration et à un premier inventaire de plus de 3000 clichés photographiques par Josef Tcherv. Au chercheur, ce travail de publication demande des efforts considérables, notamment dans la transcription (les « copies-lettres » sont parfois quasiment indéchiffrables) et le recoupement chronologique auquel Ritter déjà âgé renonça lui-même lorsqu'il dicta une « biographie » à Josef Tcherv (Ritter-Tcherv 1958 et inédits) ; au curieux, il apporte une matière de référence dans les domaines respectifs de la musicologie et de l'histoire de l'art, dont les littératures respectives ne citaient jusqu'à présent le nom de Ritter que de façon aléatoire.

Mais peut-on briser le syndrome d'Eckermann ? Autrement dit, peut-on dépasser cette approche exclusivement disciplinaire du personnage Ritter, tenter de le comprendre non seulement comme faire-valoir de tel grand personnage ou comme témoin utile à la connaissance de telle matière, mais pour soi ? Ritter fut un touche-à-tout, parfois un dilettante ; un critique, parfois péremptoire ; la diversité de ses voyages, connaissances, ouvrages et articles (projetés, avortés, achevés) et contacts font de lui un kaléidoscope oublié sur lequel il vaut la peine de coller son œil : car il imposa, avec une franchise qui allait parfois jusqu'à l'ingénuité, certaines dissonances dans la culture helvétique et en général francophone de la « fin de siècle ». Arrêtons-nous ici sur quelques-unes d'entre elles, toutes motivées par une quête d'authenticité : c'est elle en effet qui le lança vers des horizons méconnus, des pays des Balkans puis de l'Europe centrale, le poussa à les évoquer d'une plume singulière et lui permit d'y cultiver ses différences, entre autres son amour des garçons. Il s'inscrit donc trois fois de la culture 1900 : dans l'histoire des rapports interculturels, du style, mais aussi de l'éveil de la question des « genres ». Évoquons ici tour à tour le voyageur, le styliste et l'homosexuel.

\section{«Sur les grands chemins du monde»}

Il existe deux photos, respectivement de William Ritter (fig.I) et de Janko Cádra (fig.2), prises dans un train (manifestement, les deux compagnons se sont mutuellement photographiés l'un après l'autre). De Janko - tête appuyée contre le rebord de la fenêtre - on ne voit presque rien du visage, le cliché original a mal vieilli et il est hachuré de rainures horizontales; William, 


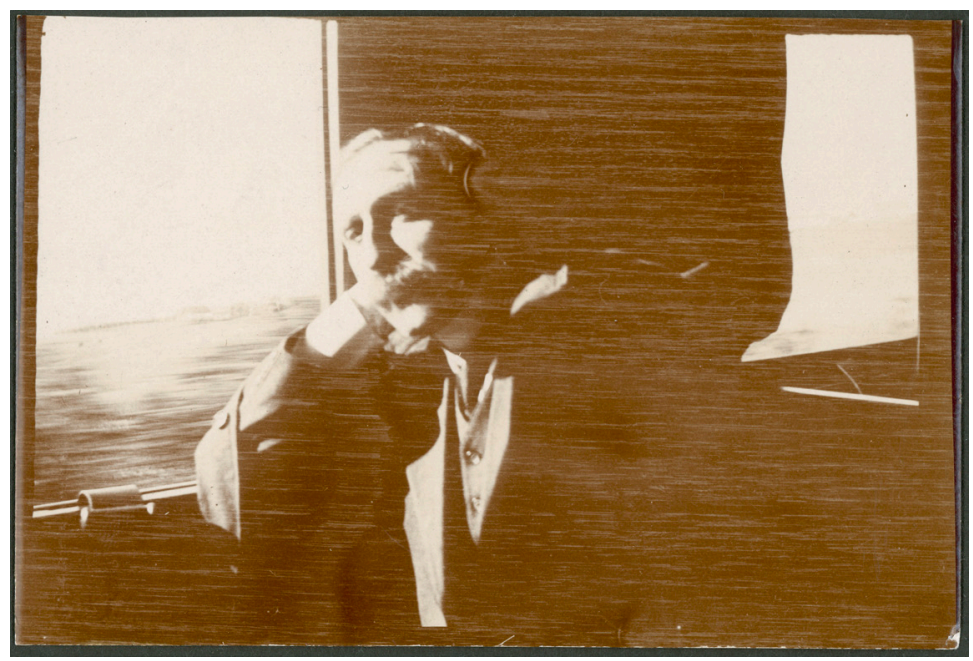

Fig. I: Janko Cádra (?), William Ritter dans le train, vers 1908. Photographie (épreuve originale), format 9 × $6 \mathrm{~cm}, \mathrm{BPUN}$ (WR-CARTo3-POS-I55).

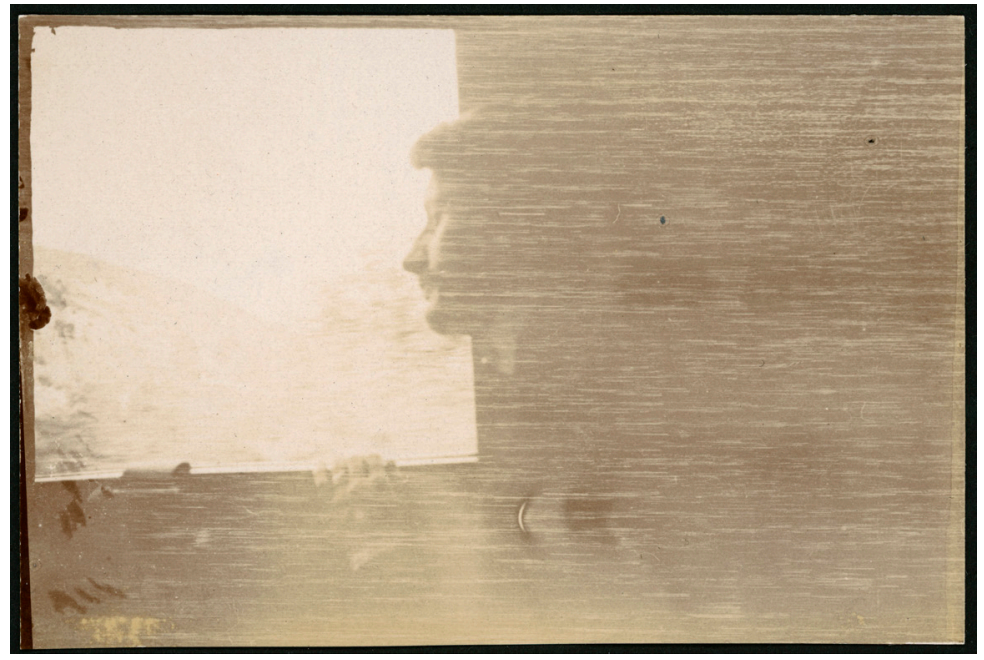

Fig. 2: William Ritter (?), Janko Cádra dans le train, vers 1908. Photographie (épreuve originale), format $9 \times 6 \mathrm{~cm}$, BPUN (WR-CARTo3-POS-I57).

mieux visible, est renversé sur la banquette comme sous l'effet du paysage qui défile, ce que la photo laisse imaginer par le flou de l'arrière-plan : image alerte d'un voyageur dont toute la curiosité s'assouvit par la grande 
fenêtre 3 . Ritter fut l'homme d'innombrables équipées à l'Est - en train, donc, mais parfois aussi à pied..., qui lui inspirèrent des écrits (il prétendait d'ailleurs en faire son gagne-pain) dont les titres parlent d'eux-mêmes : Bucharest pendant la semaine sainte (I890), Correspondance d'Autriche (I894), La nation tchéco-slovaque (I894), Second voyage au Monténégro (1895), Heures de Prague et de Vienne (I896), L'art décoratif en Autriche-Hongrie (I897), Bilan de l'art polonais (1908), Carnet munichois (I9I3)... On pense à les lire à cette expression $\mathrm{du}$ journaliste puis romancier autrichien Karl Emil Franzos qui traitait de « semi-Asie » les confins orientaux de l'empire austro-hongrois. À ceci près qu'il s'agit exactement de l'inverse : Juif de Galicie, Franzos avait, en faisant carrière, assimilé le point de vue impérial et les visées colonisatrices viennoises sur les contrées quasi-sauvages de ses franges Est. «Halb-Asien » : des paysages immenses où commence la steppe, aux chemins crottés, dont les populations arriérées végètent en attendant les lumières - le savoir et l'école tout comme l'électricité. C'est la même différence civilisationnelle que contemple Ritter, mais dans l'autre sens, en jouissant de la « semi-barbarie " des régions découvertes dont il tente de partager les idéaux et les tourments (Galmiche 20I2). Dans cet entre-deux, qui mêle l'exotisme et la familiarité, se forge un regard qui est une sorte de double inversé du colonial: dans la logique de l'empathie, Ritter donne a priori et indéfectiblement la préférence aux réalités indigènes. Cette compassion au sens originel l'entraîne parfois à des contradictions majeures : d'une part, il adopte aveuglément les passions de ses amis, que sont, dans le contexte centre-européen de l'époque, l'anticentralisme ardent des peuples de l'Empire, notamment contre Budapest, et l'antisémitisme local (Szabó 2008 ; Galmiche 20II ; 2019 : 83-9I). D'autre part, il affectionne par principe ce que la rumeur et la critique semblent avoir le moins consacré (c'est une raison mentale, en plus des motifs affectifs et esthétiques, d'aimer la Slovaquie, par exemple - Xavier Galmiche (20I9 : I23-I3I) ; c'est aussi le ressort de son antimodernisme, flagrant à partir des années I900), et cette affection le pousse à écrire : paradoxe insoluble, car plus il s'en explique, plus il la sape.

\section{Un styliste}

La diversité des genres littéraires pratiqués par le voyageur journaliste écrivain que fut Ritter est extrême : articles, monographies, recueils d'impressions de voyages, essais, romans. On sait que Ramuz a exprimé nettement la répulsion que lui inspirait ce « Neuchâtelois slave, mieux sla-

3 Pour les photographies prises par William Ritter et ses compagnons principalement à partir de 1902, voir les clichés légués à la Bibliothèque publique et universitaire de Neuchâtel par Josef Tcherv (environ 3200 pièces, I250 épreuves originales et I500 négatifs), https://floraweb. ne.ch/flora/servlet/LoginServlet?profile=anonymous\&success=jsp/system/win_main.jsp. 
visé ! ${ }^{4}$ : pour lui, il était l'incarnation de la transgression de la latinité, puisqu'il cherchait l'authenticité du côté de l'« Europe slave » (en fait la mosaïque des peuples d'Europe centrale). Mais j'ai idée que cette aversion était aussi question de goût, car le style de Ritter est, à l'opposé de la robustesse $\mathrm{du}$ « français de plein air » qui caractérise l'auteur de Derborence (Mahrer 2006), un maniérisme sorti de l'alambic symboliste : n'importe quelle page d'ÆEgyptiacque l'atteste, pleine de préciosités, voire d'enflures, servies par des raffinements de syntaxe et, çà et là, de quelques vulgarismes. Un exemple entre mille, extrait du témoignage que Ritter écrivit en 1936 en l'hommage du peintre tchèque Miloš Jiránek, qui fut son meilleur ami au début des années I900 (Ritter 1936). Ritter lui y reproche de jouer au « peintre difficile » : d'obéir « au mécontentement opiniâtre de lui-même par tout ce qui n'était pas l'orgueil d'un idéal difficile, l'orgueil de se sentir exceptionnel, l'orgueil de se sentir, de devenir un "peintre difficile" comme il y a des "auteurs difficiles" »- mais cet éloge de la simplicité passe par une écriture pleine de raccourcis, d'allusions, de clins d'œil :

Il [Jiránek] trouvait chez nous du Grigoresco. Le vieux maître roumain eût pu lui apprendre, car les échantillons que nous avions de son art étaient largement suffisants à en retirer toute la leçon que comportait cet art [...] eût pu, eût dû lui enseigner merveilleusement ce qu'il ne voulait pas savoir, en dépit de l'évidence, que l'on peut être un merveilleux artiste, un infini poète, en s'attablant avec aisance et appétit devant ce qu'offre chaque jour notre entourage. Il voulut pourtant essayer... longtemps après, en cachette, une fois tout seul. Mais encore cum grano salis, de sel de difficulté, de contournement, de position incommode. Et l'essai demeura unique : certain arbre de la campagne de Třeboň où l'intention est nettement avouée, se souvenir de Grigoresco. Il est clair que Jiránek n'était pas né pour s'en accommoder! Il faut être né heureux. Être né peintre ne suffit pas ! (Galmiche 2019: 220).

Il faut avoir la sagacité d'apprécier ce kitsch-là.

Mais il y a autre chose: les textes de Ritter qui sont aujourd'hui peut-être les plus intéressants sont ceux qui, à l'époque, échappèrent à une insertion dans un cadre éditorial régulier et donc aussi à l'emprise de l'idéologie, de la censure, etc. À mon goût, les plus frais, presque tous inédits, relèvent de l'« écriture du moi ", journaux et calepins de voyage (nous les avons classés sous la rubrique d'" égo-histoire » dans la nomenclature de l'inventaire en cours du fonds Ritter des Archives littéraires de la Bibliothèque nationale suisse à Berne), et dans les lettres. Notons que Ritter élargit ces genres de notations non verbales, dessins ou photographies : des milliers de crayons,

4 Lettre de Charles Ferdinand Ramuz à Adrien Bovy [Paris, juin 1905], in Guisan, Gilbert, C. F. Ramuz, ses amis et son temps, t. 2, lettre 249, Lausanne, Bibliothèque des arts, 1967. Cité par Michaud (2008: 8). 
fusains ou pastels, reflétant au jour le jour des scènes intimes de la vie familière (par exemple et surtout, infiniment, Janko croqué dans des positions à teneur érotique : " au tub du matin », nu cirant des chaussures, nu lisant, etc.). Ajoutées les unes aux autres, notes scripturales et notes plastiques constituent une sorte de grand commentaire « en temps réel » de la vie qui passe et où tout est important, une entreprise vouée à rester confidentielle (le journal n'est pas écrit pour être publié) mais dont le caractère novateur semble ne pas avoir échappé à son auteur : Janko Cádra note dans son journal le 6 février I92I les propos de «Wilko » : «Écrire consciencieusement, simplement, sincèrement sa vie au jour le jour - c'est une œuvre très grande, une œuvre variée, vraie et durable. Et je crois qu'il n'y a pas d'exemple au monde d'un Journal comme le nôtre. » (Cádra I92I : 9). Ces pages discrètes fournissent un programme d'édition à venir, la bonne part (la meilleure ?) de cette écriture restant à extraire des centaines de milliers de pages, manuscrites ou dessinées. Je reproduis ici pour le plaisir et pour l'exemple une page du calepin du voyage en Slovaquie en I921, compte rendu prosaïque d'un dimanche comme un autre, où des remarques politiques (pas très correctes, car Ritter s'y montre déjà rétif à l'euphorie républicaine officielle qui caractérise la jeune Tchécoslovaquie) cohabitent avec la poésie d'un quotidien rythmé par la piété protestante :

Rêvé de Rupprecht de Bavière. $\Delta \tau \varepsilon$ [les notations érotiques sont cryptées en caractères grecs. $\Delta \tau \varepsilon$ indique une « détente » de Janko] $7[\mathrm{~h}]$ moins $1 / 4$. Dans le bruit des cloches tellement amaigri depuis la guerre. Et l'heure qui sonnait entière à chaque quart d'heure a totalement cessé de sonner. Et du fer blanc a remplacé au bulbe le beau cuivre vert de jadis. Déjeuner précédé de l'ordinaire stamperl [un verre de gnôle]. Remontons moi écrire ceci, Janko humecter le pansement de Joseph. Un vilain gris douteux au ciel. [...] Dehors au milieu des kopanitchiari [paysans habitants des kopanice, fermes isolées du pays de Myjava] chez l'ignoble pharmacien madyaron [traître à la cause nationale au profit du pouvoir hongrois, renégat], tout en clous et scrofules semble-t-il acheter un remède quelconque pour mes bobos aux pieds. Monument Štefaník [Rastislav Štefánik, ingénieur slovaque, engagé comme aviateur dans l'armée française, où il milita pour la création de la Tchécoslovaquie, mort en I9I9 dans un accident d'avion qui reste inexpliqué] décidément une baraque de planches et de caisses de pierre, un théorème de géométrie fort compliqué qu'aurait voulu résoudre un singe, du bolchevisme en plus. Janko nous entraîne à l'église « seulement une minute ». Mais il y reste, y a une crise de larmes, me met dehors avant la fin du chant et rentre pour le sermon, concession à son cousin. Son beau frère est à l'orgue, qui n'a que deux registres. Trois belles voûtes arrondies en large comme en long de l'orgue à l'autel, une encore derrière l'autel. Taillées comme dans de la brindza [fromage blanc de brebis]. En bas tout ce parterre de coiffes blanches plus frais qu'un champ de marguerites ou d'ombelles. Les jeunes filles debout derrière l'autel et rangées par kopanice, 
les femmes dans les bancs, les hommes aux galeries. Un papillon s'égare sur toutes ces coiffes florales. Joseph sorti le premier se promène avec Douchan. La poussière se lève du pavé raclé de terre et de temps en temps le bruit si spécial d'un char de kopanitchar qui se trimbale et grésille comme un sac de clous. Je n'arriverai jamais à travailler dans cette chambre. Aucun article d'ailleurs sur ni chants ni Bertramka-Mozart [Mozart séjourna à Prague dans la villa Bertramka] ni fête de Kyjov [localité morave célèbre pour ses rencontres de musique folklorique] et leurs démêlés tchécoslovaques me coupent les ailes. Rejoins Douchan et Joseph dehors. Tour par derrière la synagogue et la stara rjeka [en slovaque : vieille rivière] jusqu'à l'église catholique que nous trouvons fermée. [...] Le dîner nous est servi à midi. [...] A deux heures chez nous sieste. Et voici les belles grosses cloches du culte d'après-midi. Pétition des gens de la ville qui ont demandé un culte plus court. Les kopanitchari se sont révoltés. Nous ne faisons pas deux heures de chemin aller et retour pour ne prier qu'une $1 / 2$ heure. Ils en veulent pour leur argent (Ritter I92I, f. I3).

\section{L'adorateur}

Enfin (et pour certains : surtout), Ritter étonne par l'insistance avec laquelle il témoigne de son amour pour les garçons. Motif dominant de l'égo-histoire, celui-ci aboutit à une homosexualité identifiée, assumée et vécue, toute la vie durant, et parfois dans le bonheur.

Il y eut certes des conditions favorables : des parents plutôt libéraux (les photos où le père, Guillaume Ritter, pose avec le couple William-Janko sont nombreuses) et une époque propice. Le monde littéraire de la génération décadente poussa William à affirmer sa différence sexuelle : Barbey d'Aurevilly à qui il écrivit, Joséphin Péladan qui le conseilla en la matière à « aller de l'avant, dandyment » et, à sa suite, Carmen Sylva puis Pier re Loti (Galmiche 2008). L'homosexualité motive le culte d'Arthur Rimbaud et quelques amitiés littéraires, avec Robert de Montesquiou (voir Ritter I893) et surtout Stefan Georg (Ritter I895).

Mais l'éloignement du lieu d'origine fut le véritable déclencheur de cette émancipation : loin de ces coteries, la vie véritable se tissa avec des garçons rencontrés au hasard des voyages. Parmi les nombreux hommes qui l'éblouirent, trois partagèrent sa vie, un Français rencontré en Roumanie, Marcel Montandon, le Slovaque Janko Cádra et le Tchèque Josef Červ. Le journal et ses cahiers érotiques («Pages secrètes »), les lettres et les photos donnent l'image d'une homosexualité sans tabou : s'imposant dans les milieux de Munich, Vienne et Prague en compagnie d'amis qui, pour la plupart, entretiennent l'amitié entre garçons avant de faire des mariages bourgeois, Ritter cultiva, à partir de sa liaison avec Janko, une vie en couple qui semblait imiter le modèle conjugal hétérosexuel, mais l'accommodait de nombreux arrangements. D'abord par l'idéal pédérastique grec, puisqu'il se 
voyait en formateur des garçons qu'il aimait et entourait ; ensuite, par l'idée du bonheur dans le couple à trois. Il faudrait s'attarder sur les années entre I900 et la guerre où, dans le monde fluide de la culture munichoise, William transforma ces penchants en mode de vie, s'installant d'abord avec Janko dans un appartement qui jouxtait celui de Marcel désormais marié et père de famille, puis attira Josef dans ce nid conjugal.

Ritter synthétise de nombreux emblèmes de la subculture homosexuelle telle qu'elle s'est développée depuis la fin du XIX ${ }^{\mathrm{e}}$ siècle, depuis la musique de Wagner et Mahler au culte pour les images de saint Sébastien, la pratique du « journal de drague » où sont notées les rencontres plus ou moins abouties, et, plus spécifique il est vrai, le goût pour les Pages secrètes, des chapitres thématiques sur des matières pornographiques au sens strict, comme $\Phi \alpha \lambda \lambda$ os ou Beauté, ode à la chair virile.

[...] Hadrien déïfia Antinoüs. Il suffit pour cela de le sculpter nu.

Je voudrais que tous les amants consentissent à se deïfier mutuellement. Et pour ma part on saura quelque jour de quelle façon je m'y suis efforcés.

[...] Il [Le Phallus] est aussi expressif que le visage et il ne sait pas mentir. Le portrait véridique de tout homme pourrait se faire par le phallus. Il raconterait la vie de cet homme autant que sa tête.

[...] L'érection, l'air de tête du coq sexuel subtilement redressé et attentif-le coup de «l'ergot du sexe " comme a dit Suarès - la subite présence d'une corne au ventre - le " ventre cornu » est de Bonaventure Desperiers ${ }^{6}$ - quatre mots latins, quatre mots lapidaires - ils sont de Pétrone - l'expriment plus plastiquement - d'une plasticité à valeur d'onomatopée - que tout ce qui a jamais suivi :

"Quod antea jacebat - stat »

C'est la perfection, c'est l'absolu, c'est la statue et c'est l'acte.

M. Macedonski n'a plus qu'à offrir son héroïne qui deviendra le « vivant

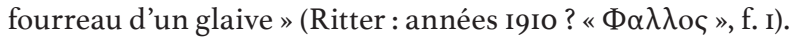

\section{Conclusion}

Voyageur, esthète, amoureux des garçons : sans doute ces aspects sontils complémentaires dans l'atmosphère du début du Xx siècle. Il n'empêche qu'ils font de lui un mélange original et un peu détonnant, un personnage kaléidoscopique que nous serons, Suisses ou non, bien inspirés de cesser de méconnaître.

5 Probable allusion à ses croquis de nus.

6 Nouvelles récréations et joyeux devis de Bonaventure des Périers (1558). 


\section{Bibliographie}

Bourgarel, Gérard, Tomczak, Grzegorz \& Pasquier, Augustin, Józef Mehoffer: de Cracovie à Fribourg, ce flamboyant art nouveau polonais, Pro Fribourg, Repères fribourgeois 7, 1995.

De Janko Cádra, «Deník » [Journal] I9I7-I923 (Slovenská národná knižnica [Bibliothèque nationale slovaque] - Literarný archiv, $55 \mathrm{~S}$ 52), 820 p., Ms, $\mathrm{I}^{\text {ère }}$ liasse.

Galmiche, Xavier, " "Tout ce qui de mon temps donnait sa physionomie particulière à l'Autriche-Hongrie tout entière." William Ritter en Slovaquie : le syndrome empathique ", Cultures d'Europe centrale. Hors série. "Miroirs brisés. Récits régionaux et imaginaires croisés sur le territoire slovaque ", $\mathrm{n}^{\circ}$ 7, Paris, CIRCE / Institut d'études slaves, 201I, pp. 205-216.

—. «William et les garçons (d'Europe centrale) : "Aventures de volupté sous d'autres cieux" ", plateforme William Ritter, http://www.circe.parissorbonne.fr, 2008.

—. "William Ritter et les "façons boyaresques" de la "vieille Roumanie", I890 - Syndrome empathique, fascination pour les confins et identification au semi-barbare », in "Halb-Asien " und Frankreich. Erlebtes und erinnertes Osteuropa in Literatur und Geschichte, éd. Charlotte Krauss, Ariane Lüthi, Berlin, Lit Verlag, 20I2, pp. 9I-IO4.

-. William Ritter voyage en Slovaquie (I903-I9I4) - Images d'un pays rêvé, II tomes, Paris, Eur'ORBEM Éditions, 2019.

Galmiche, Xavier, Markéta, Theinhardt, « Joža Uprka a William Ritter » in Joža Uprka (I86I-1940) : Evropan slováckého venkova, catalogue de l'exposition Valdštejnská jízdárna 23.9.20II-22.I.20I2, éd. Helena Musilová, Prague, Národní galerie v Praze pro Nadaci Moravské Slovácko, 2OII, pp. I77-I9I.

Kirschbaum, Stanislav J., Slovaques et Tchèques. Essai sur un nouvel aperçu de leur histoire politique, préface de Georges-André Chevallaz, I987; Renée Perréal et Joseph A. Mikuš, La Slovaquie, une nation au coeur de l'Europe, préface de J.-M. Rydlo, Lausanne, L'Âge d'homme, « Centre des études slaves William Ritter ", 1992.

Le Corbusier, William, Ritter, Correspondance croisée I9IO-I955, éd. Marie-Jeanne Dumont, Lettres à ses maîtres / Le Corbusier 3, Paris, Éditions du Linteau, 2014.

Mahrer, Rudolf, «Un français de plein air : la langue romanesque de Ramuz », Le Français moderne, no 2, 2006, pp. 219-235

Meylan, Claude, William Ritter chevalier de Gustav Mahler. Écrits, correspondance, documents, Bern - New York, Peter Lang, 2000.

Michaud, Marius, «William Ritter vu par ses contemporains suisses : "amateur d'Europe centrale" et contempteur de son pays natal ", in Actes du colloque "L'Europe centrale en amateur. William Ritter (I867-I955)", 
2I-22 novembre 2008, Paris, Centre interdisciplinaire de Recherches centre-européennes, Plateforme William Ritter, http://www.circe.paris-sorbonne.fr.

Ritter-Tcherv, Josef, Enfance et jeunesse I867-I889, Melide, Éditions de la Flèche d'or 1958; « Maturité I890-I9I4 » et «Vieillesse I9I4-I955 », Ms, ALS, Fonds William Ritter.

Ritter, William, "Les Chauves-souris du Comte Robert de Montesquiou-Fezensac ", Le Magasin littéraire, Io mai 1893, p. 40I.

—. «La poésie allemande nouvelle. M. Stefan George », La Semaine littéraire, 2 mars I895, n' 6I, pp. 97-99.

—. «Pages secrètes », Ms, ALS, Fonds William Ritter, Boîte 69, Cahier A, années I9IO (?).

—. La Passion de l'art en Moravie : notes de voyage (été 1908), extrait de la Bibliothèque universelle et Revue suisse, Lausanne, 1912.

Szabó, Miloslav, " "The Victory of Swindle over the Folk's Beauty". The Aesthetical Populism and Anti-Semitism of Ján Cádra », in Actes du colloque "L'Europe centrale en amateur. William Ritter (I867-I955)", 2I-22 novembre 2008, Paris, Centre interdisciplinaire de Recherches centre-européennes, Plateforme William Ritter, http://www.circe.paris-sorbonne.fr.

Utopierre: Guillaume Ritter, de Vieux-Châtel à Neuchâtel, Jean-Baptiste Cotelli [et al.] : textes réunis par Nicole Bauermeister, préf. de Jacques Bujard, photogr. de Reno Sterchi, Le Locle, éditions G d'encre, 2009.

Vlasiu, Ioanna, "Rencontre Nicolae Grigorescu-William Ritter. Perspectives des historiens de l'art », in Actes du colloque « L'Europe centrale en amateur. William Ritter (I867-I955) », 2I-22 novembre 2008, Paris, Centre interdisciplinaire de Recherches centre-européennes, Plateforme William Ritter, http://www.circe.paris-sorbonne.fr.

Zabu, Wahlen, Roulent leurs eaux à contretemps, Carnets de voyage I893-20I3, Lausanne, art\&fiction, coll. « Re:Pacific », 2016.

\section{Textes inédits}

Calepin de voyage I92I, ALS, Fonds William Ritter, Boîte 56.

« La jeunesse de Miloš Jiránek. Souvenirs des années I896 à I906 », I936, version française inédite, ALS, Fonds William Ritter, Boîte 4I ; traduction tchèque : "Mládí Miloše Jiránka ", in Catalogue de l'exposition Neznámý Miloš Jiránek [Miloš Jiránek inconnu] Salon Topič, Prague 19 XI-8 XII 1946, précédée d'une lettre du 2 novembre 1946, pp. 4-27.

Ritter, William, « Pages secrètes », Ms, ALS, Fonds William Ritter, Boîte 69, Cahier A, années I9Io (?). 
De Janko Cádra, « Deník » [Journal] I917-I923 (Slovenská národná knižnica [Bibliothèque nationale slovaque] - Literarný archiv, $55 \mathrm{~S}$ 52), 820 p., Ms, $\mathrm{I}^{\text {ère }}$ liasse.

"Plateforme William Ritter » sur le site du CIRCE (Centre interdisciplinaire de Recherches centre-européennes), http://www.circe.paris-sorbonne.fr, lien direct http://www.circe.paris-sorbonne.fr/index. php?option $=$ com_content\&view $=$ article \&id $=204 \% 3$ Awilliam-ritter-introduction\&catid=50\&Itemid=I3. $S$ y trouvent édités des textes suscités par le colloque «Europe centrale en amateur. William Ritter (I867-1955) » qui eut lieu en 2008; contributions et documents peuvent y être ajoutés. Ritter-Tcherv, Josef, « Maturité I890-I9I4 » et «Vieillesse I9I4-I955 », Ms, ALS, Fonds William Ritter. 